\title{
ORIENTATION EFFECTIVENESS IN THE OBJECTS DETECTED AREAS USING DIFFERENT TYPES OF EDGES DETECTION TECHNIQUES
}

\author{
Muhamad Azhar Abdilatef AL-Obaidy \\ Department of Mechatronics Engineering, College of Engineering, University of Mosul, \\ Mosul, Iraq
}

\begin{abstract}
This paper presents a study for the orientation effectiveness on the detected areas for many sampled objects when many type of the edges detection are applied. The Canny, Laplace, Prewitt and Sobel are applied for three objects (pencils' sharpeners with different colors). The MBR (Minimal Bounding Rectangular) are used to calculate the area in pixels, centroid and the orientation. The MR (Misclassification Ratio) is used to find the different between the edges detection techniques. The Canny edges detection technique gives the best result for the three used object using all orientations.
\end{abstract}

\section{KEYWORDS}

Orientation, Edge Detection, MR, Objects, tp rate, sharpeners, Canny, Sobel, Laplace, Prewitt.

\section{INTRODUCTION}

The object detection technique is one of the most important image processing and computer vision methods those used in many fields today. During the detection many features can be extracted such as colors, orientations, centroids, textures, edges, and ext. the detection methods and especially the edges techniques are used in the fields of military, medicine, biological researches, industrial applications and many others. These techniques are sometime used to support other types of detection methods, it can help them to complete the final work such as the medical and the industrial applications, in other time it represent the mainly part of the work. Three different pencil sharpeners with different colors: Blue, Green, and Indigo are detected used four types of edges detection (Figure 1); the area in pixel, centroid and the orientation are calculated using the MBR, while the MR (Misclassification Ratio) is used to calculate the detection accuracy for all types of the used edges detection (Canny, Laplace, Sobel, Prewitt) when applied for these objects. According to the used methods and referring to the obtained results, the canny edges detection is the best one of the applied method.

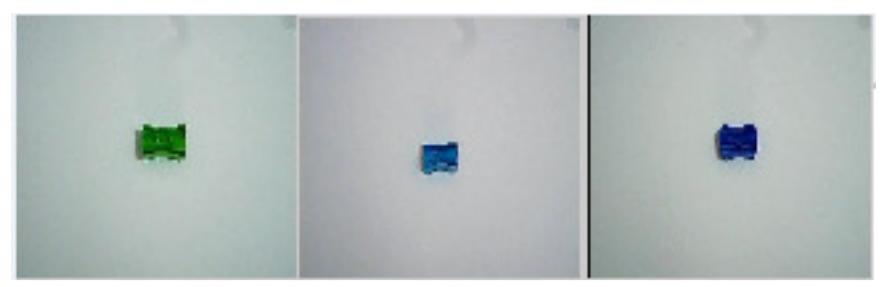

Figure 1: The samples used objects 
International Journal of Computer Science \& Engineering Survey (IJCSES) Vol.8, No.2, April 2017

\section{RELATIVE WORKS}

Canny and other types of edges detection techniques are used by the researcher inside other type of image processing detection methods in their works, as mentioned before, these methods can be used individually or with others, the canny edge detection is an operator developed by John F. Canny, it is used for purpose of detection, it uses a multi stage algorithm type [7]. The Sobel technique which is mostly named the sobel filter or sobel-Feldman operator is presented by Irwin Sobel in 1968, it is used in computer vision and also image processing detection algorithms [11], it is really a discrete differentiation technique, it can compute the gradient of the intensity function of the image, in this type, each point or pixel inside the image is corresponded as a gradient vector or norm to it. The prewitt operator [12] which is presented by Judith M.S. Prewitt is found the edges using the prewitt approximation to the derivative. In most of the works today, many proposed methods merged between two or more methods to present new techniques with many enhancements, other researches are done depending on a comparison between many methods over the same material (objects). In [1], Min. C. Shin and et.al a comparison between the edges detection techniques is done. They used the structure from motion (SMF) to do an evaluation over the algorithms of edges detections. The obtained results suggested that the ratings of the canny detector performance is based on the pixel level matrices and also on the SFM are related one to each other and have high performance like canny and other detectors. In [2], Sayed Jahanzeb Hussien Pirzada and et.al are enhanced the edges detection techniques using low pass filter inside the canny edge detection, this algorithm is used as a features extraction, the extracted features are not same when its captured from different directions. In this work, a study of an orientation's effectiveness for the detected objects is done, assumed that the objects images are captured from many sides; many edges types are applied for these images with which are content same shape objects with different colors. In [3], Andrew H. S Lai, and Nelson H. C. Yung are presented a novel method for lane algorithm detection used for surveillance traffics tracking, they tried to isolate one path from all the captured image then surrounding or mask the car needed to be detected or tracked from the other parts, also used to notes any sudden changes or movements, the edges techniques are used here and the orientation is detected during the detection and tracking tackle, most of the time when the objects are detected more one time with many orientations, the shapes and also number of pixels those extracted are not the same. Another study for the orientation inside the colors is done by Viswanath Gopalakrishnan, Yiquan $\mathrm{Hu}$, and Deepu Rajan [4], they used the detection framework which is based on both color and orientation those distributed in image, the orientation framework is used to detect regions in images based on global and local behaviour of different orientations in images. In robotics field when an objects sometime has to be gripped by the fingers of the robots, it is very important to know the orientation of it to rotate the robot arm according to that and catch it, In [5], a study for a mobile cleaning robot is done to measure the error in orientation, a new method is used to reduce the error in localization. In this paper, after studied the ideas given before, a new study for the orientation effectiveness is used to compare between many edges detection techniques when the same regions are detected from different sides, this rule is applied for the areas of detected objects used these many edges techniques.

\subsection{Canny Edge Detection Technique}

The canny edge detection is an operator or technique used for extracting the useful information and structures details from different vision's object, canny and the other type of edges techniques can reduce the amount of data to be processed. The canny algorithm includes applying the Gaussian filter which is used to smooth the image to remove the noisy parts, find the image gradients' intensity, applying the non-maximum type of suppression for the purpose of liquidation for the response's spurious to the edge detection, duplicate the normal value of threshold and finally using the hysteresis to track the edge [7]. 


\subsection{Sobel Edge Detection Technique}

Sobel filter or as it is sometime called Sobel- Feldman operator, is a type of edges techniques used in image processing and also in computer vision, this type consist of two separated operations: the first one make a smoothing to be perpendicular to the direction that is derivative with a triangle filter, the second one is the central simple difference in the derivative direction, the Sobel operator can reduce the artifacts that is associated with the pure central operator's differences, but it does not have a perfect rotational symmetry. So this is a disadvantage of using it [8].

\subsection{Laplace Edge Detection Technique}

The Laplace detector or as it is named the Laplacian operator is mostly represented as a blob detector that is used in computer vision field for the purpose of regions detection in digital captured images from a real or offline video (recorded video), this type of operators is used in this paper as one of many techniques applied for the purpose of orientation effectiveness.

\subsection{Prewitt Edge Detection Technique}

It is a discrete differentiation technique or operator that includes computing the gradient approximately for the image intensity function, this operator is usually based on convolving the used image with a separable, small, and integer filter value in both horizontal and vertical directions, it like Sobel operator in that it is inexpensive in computations relatively.

\section{WORK AND RESUltS}

In this paper, canny, Sobel, Prewitt, and Laplace edges detection methods are applied for three colors of a pencil sharpener (light blue - dark blue - green), each of these three sharpeners are captured in many orientations: $(45 \mathrm{o}, 90 \mathrm{o}, 135 \mathrm{o}, 180 \mathrm{o})$, figures $(2-4)$, includes the area in pixels which are detected for three sharpeners with all the four mentioned orientations after applying the edges. The MBR is used to get the wanted orientation exactly, The purpose of this operation is to see if the changes in orientations may cause effects in detection (the edges techniques) or not, and to see also which type of edges techniques has the less effectiveness when the orientation is changed, the following tables ( 1 - 4) are included the areas detected using the edges detection techniques for the sharpeners in all orientations cases, table 5 includes the correctly calculated areas before applying the edges techniques.

Table $1.45^{\circ}$ orientation objects detected results

\begin{tabular}{|l|l|l|l|}
\hline & Object1 & Object2 & Object3 \\
\hline CANNY & 1313 & 946 & 1458 \\
\hline SOBEL & 1309 & 942 & 1466 \\
\hline PREWITT & 1307 & 944 & 1471 \\
\hline LAPLACE & 1305 & 938 & 1466 \\
\hline
\end{tabular}

Table $2.90^{\circ}$ orientation objects detected results

\begin{tabular}{|l|l|l|l|}
\hline & Object1 & Object2 & Object3 \\
\hline CANNY & 1363 & 885 & 1451 \\
\hline SOBEL & 1346 & 882 & 1460 \\
\hline PREWITT & 1359 & 883 & 1456 \\
\hline LAPLACE & 1349 & 871 & 1455 \\
\hline
\end{tabular}


International Journal of Computer Science \& Engineering Survey (IJCSES) Vol.8, No.2, April 2017

Table $3.135^{\circ}$ orientation objects detected results

\begin{tabular}{|l|l|l|l|}
\hline & Object1 & Object2 & Object3 \\
\hline CANNY & 1339 & 913 & 1445 \\
\hline SOBEL & 1328 & 907 & 1439 \\
\hline PREWITT & 1327 & 903 & 1448 \\
\hline LAPLACE & 1325 & 904 & 1438 \\
\hline
\end{tabular}

Table 4.180 orientation objects detected results

\begin{tabular}{|l|l|l|l|}
\hline & Object1 & Object2 & Object3 \\
\hline CANNY & 1351 & 996 & 1397 \\
\hline SOBEL & 1344 & 992 & 1397 \\
\hline PREWITT & 1340 & 990 & 1355 \\
\hline LAPLACE & 1335 & 981 & 1367 \\
\hline
\end{tabular}

Table 5. Correctly calculated object's areas

\begin{tabular}{|c|c|c|c|}
\hline $\begin{array}{c}\text { Orientati } \\
\text { on }\end{array}$ & OBJ1 & OBJ2 & $\begin{array}{c}\text { OBJ } \\
3\end{array}$ \\
\hline $45^{\circ}$ & 1311 & 945 & $\begin{array}{c}147 \\
2\end{array}$ \\
\hline $90^{\circ}$ & 1360 & 884 & $\begin{array}{c}146 \\
5\end{array}$ \\
\hline $135^{\circ}$ & 1334 & 912 & $\begin{array}{c}144 \\
9\end{array}$ \\
\hline $180^{\circ}$ & 1348 & 994 & $\begin{array}{c}139 \\
8\end{array}$ \\
\hline
\end{tabular}
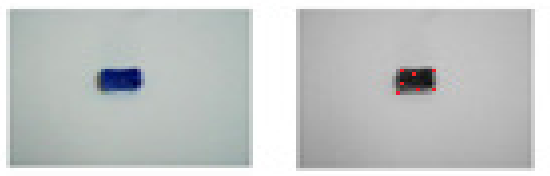

$180^{\circ}$
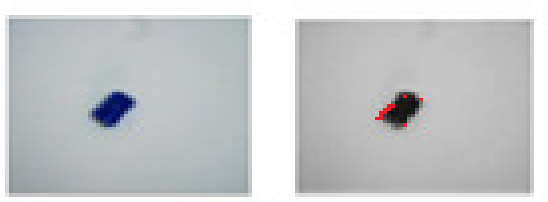

\section{$135^{\circ}$}
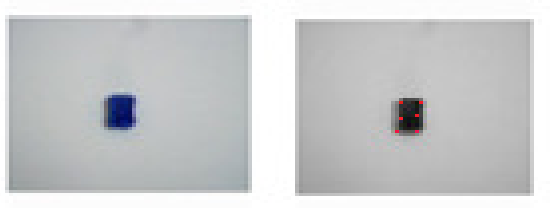

\section{$90^{\circ}$}
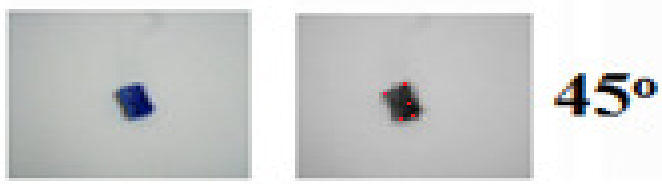

Figure 2: calculated area for object 1 
International Journal of Computer Science \& Engineering Survey (IJCSES) Vol.8, No.2, April 2017
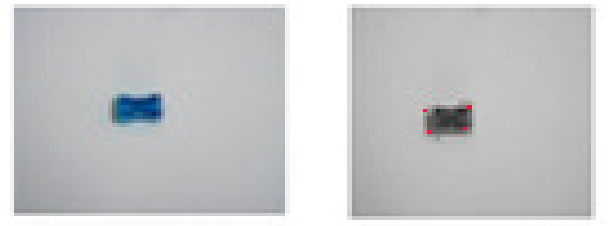

\title{
180 o
}
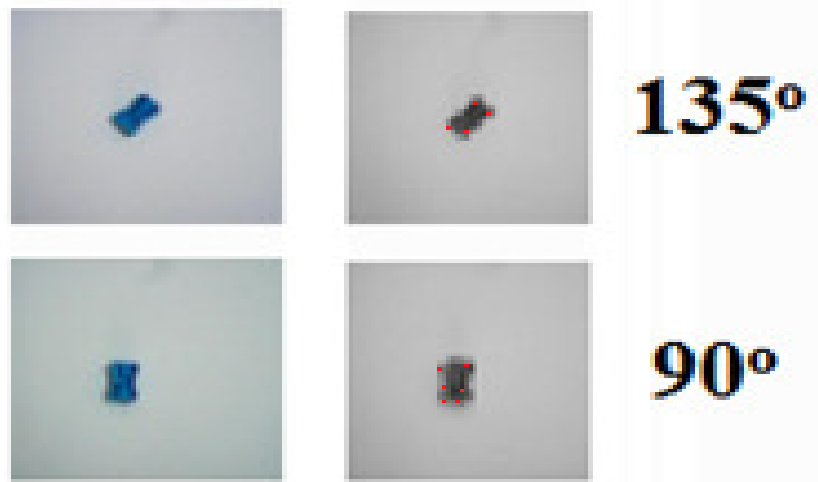

\author{
$90^{\circ}$
}
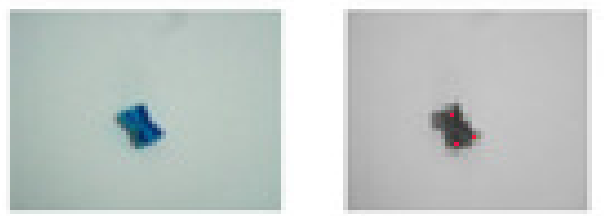

$45^{\circ}$

Figure 3: calculated area for object 2
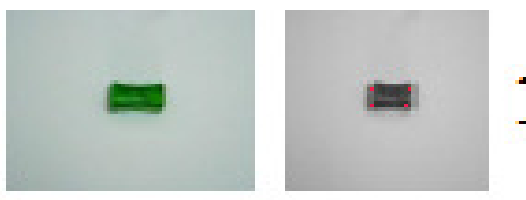

\section{$180^{\circ}$}
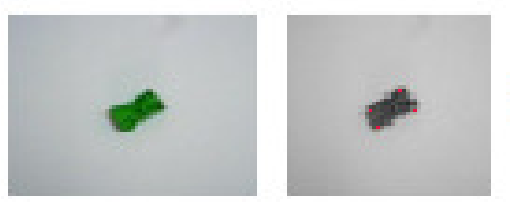

\section{$135^{\circ}$}
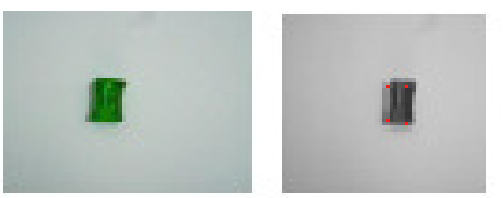

\section{$90^{\circ}$}
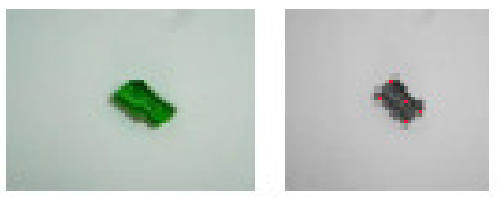

\section{$45^{\circ}$}

Figure 4: calculated area for object 3 
Sometime the number of detected pixels in the region of interest are not reach the actual foreground pixel's number thus many methods are used over the time to refer to the errors and make many enhancement for the not well detected methods, the MR (Misclassification Ratio) [910], is used to find the error in detection for each one of the three used sharpeners (objects) for the four detected orientations $(45,90,135,180)$; to do that, the correctly area of the objects has to be calculated firstly, this is done by assuming that the object (sharpener) is a foreground part while the other parts are represented as a background, thus the subtraction operation is applied for the binary images mode to calculate the correctly object's area. The following steps and equations are used to find the MR:

- Total positive numbers (foreground) pixels which are correctly classified (TP) are normalized by the total positive (foreground) in order pixels to find (tp rate).

- Total positive numbers (foreground) pixels which are incorrectly classified (FP) are normalized by the total positive (foreground) pixels in order to find (fp rate).

- Total negative numbers (background) pixels which are correctly classified (TN).

- Total negative numbers (background) pixels which are incorrectly classified (FN).

- The true positive rate [10] (also called hit rate and recall) of a classifier is estimated as:

- $\quad$ tp rate $=$ Positives correctly classified (TP) $/$ Total positives

- The false positive rate [10] (also called false alarm rate) of the classifier is estimated as:

- $\quad \mathrm{fp}$ rate= Positive incorrectly classified (FP) / Total negatives

- Finally to calculate the MR the following equation has to be used according to the following equation:

- $\mathrm{MR}=(\mathrm{FP}+\mathrm{FN}) /(\mathrm{TP}+\mathrm{TN}+\mathrm{FN}+\mathrm{TN}) * 100 \%$

Figure 5 include a flow chard to find the tp, fp and MR factors in steps. In order find all of the mentioned factors, it is important to record the information of the captured images for the used objects before applying operations, these information include the directions of image to calculate the amount of each image's pixels, table 6 include the properties for the used images.

Table 6. Used images properties

\begin{tabular}{|c|c|}
\hline Dimensions & $\mathbf{3 2 0} \times \mathbf{2 4 0}$ \\
\hline Width & 320 pixels \\
\hline Height & 240 pixels \\
\hline $\begin{array}{c}\text { Horizontal } \\
\text { Resolution }\end{array}$ & $96 \mathrm{dpi}$ \\
\hline Vertical Resolution & $96 \mathrm{dpi}$ \\
\hline Bit Depth & 24 \\
\hline
\end{tabular}


International Journal of Computer Science \& Engineering Survey (IJCSES) Vol.8, No.2, April 2017

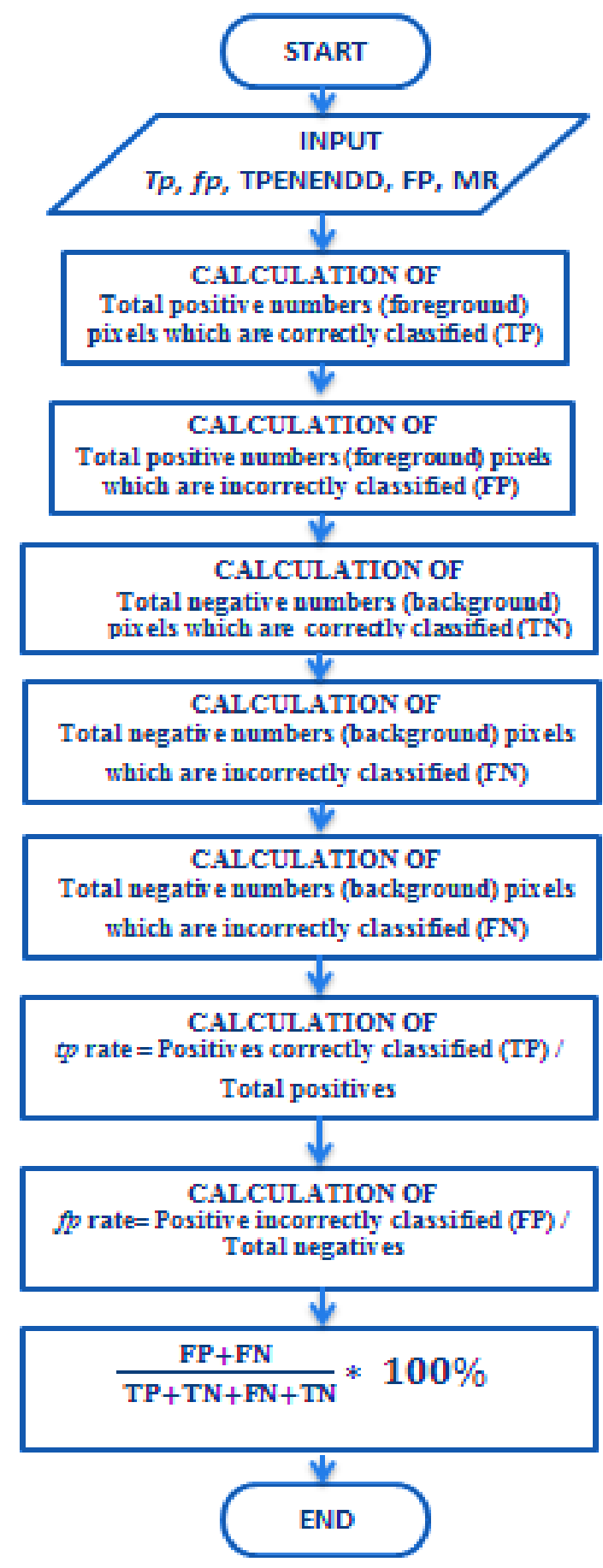

Figure 5: $t p, f p, \mathrm{MR}$ calculation

The following tables (7-15) includes the TP, FP, $t p$, MR for the all given results from when the edges techniques are applied for the used objects, the total amount of pixels for all the used images are 76800 pixels. 
International Journal of Computer Science \& Engineering Survey (IJCSES) Vol.8, No.2, April 2017

Table 7. Object $1 \mathrm{MR}$ result for $45^{\circ}$

\begin{tabular}{|c|c|c|c|c|c|c|}
\hline \multicolumn{7}{|c|}{ Result for object 1 / orientation : $45^{\circ}$} \\
\hline EDGES & TP & FP & TN & F & MR (\%) & tp \\
\hline Canny & 131 & 2 & 7548 & 0 & $26 \times 10^{-4}$ & 99.997 \\
\hline Sobel & 130 & 0 & 7548 & 2 & $26 \times 10^{-4}$ & 99.997 \\
\hline Prewitt & 130 & 0 & 7548 & 4 & $52 \times 10^{-4}$ & 99.994 \\
\hline Laplace & 130 & 0 & 7548 & 6 & $78 \times 10^{-4}$ & 99.992 \\
\hline
\end{tabular}

Table 8. Object $1 \mathrm{MR}$ result for $90^{\circ}$

\begin{tabular}{|c|c|c|c|c|l|c|}
\hline \multicolumn{7}{|c|}{ Result for object 1 / orientation : $90^{\circ}$} \\
\hline EDGES & TP & FP & TN & FN & MR (\%) & tp \\
\hline Canny & 1360 & 3 & 75437 & 0 & $39 \times 10^{-4}$ & $99.996 \%$ \\
\hline Sobel & 1346 & 0 & 75440 & 14 & $182 \times 10^{-4}$ & $99.981 \%$ \\
\hline Prewitt & 1359 & 0 & 75440 & 1 & $13 \times 10^{-4}$ & $99.998 \%$ \\
\hline Laplace & 1349 & 0 & 75440 & 11 & $143 \times 10^{-4}$ & $99.985 \%$ \\
\hline
\end{tabular}

Table 9. Object $1 \mathrm{MR}$ result for $135^{\circ}$

\begin{tabular}{|c|c|c|c|c|l|c|}
\hline \multicolumn{7}{|c|}{ Result for object 1 / orientation : $135^{\circ}$} \\
\hline EDGES & TP & FP & TN & FN & MR (\%) & tp \\
\hline Canny & 1334 & 5 & 75461 & 0 & $65 \times 10^{-4}$ & $99.993 \%$ \\
\hline Sobel & 1328 & 0 & 75466 & 6 & $78 \times 10^{-4}$ & $99.992 \%$ \\
\hline Prewitt & 1327 & 0 & 75466 & 7 & $91 \times 10^{-4}$ & $99.990 \%$ \\
\hline Laplace & 1325 & 0 & 75466 & 9 & $117 \times 10^{-4}$ & $99.988 \%$ \\
\hline
\end{tabular}

Table 10. Object $1 \mathrm{MR}$ result for $180^{\circ}$

\begin{tabular}{|c|c|c|c|c|l|c|}
\hline \multicolumn{7}{|c|}{ Result for object $1 /$ orientation : $180^{\circ}$} \\
\hline EDGES & TP & FP & TN & FN & MR (\%) & tp \\
\hline Canny & 1348 & 3 & 75449 & 0 & $39 \times 10^{-4}$ & $99.996 \%$ \\
\hline Sobel & 1344 & 0 & 75452 & 4 & $52 \times 10^{-4}$ & $99.994 \%$ \\
\hline Prewitt & 1340 & 0 & 75452 & 8 & $104 \times 10^{-4}$ & $99.989 \%$ \\
\hline Laplace & 1335 & 0 & 75452 & 13 & $169 \times 10^{-4}$ & $99.983 \%$ \\
\hline
\end{tabular}

Table 11. Object 2 MR result for $45^{\circ}$

\begin{tabular}{|c|c|c|c|c|l|c|}
\hline \multicolumn{7}{|c|}{ Result for object 2 / orientation : 45 } \\
\hline EDGES & TP & FP & TN & FN & MR (\%) (\%) (\%) & tp \\
\hline Canny & 945 & 1 & 75854 & 0 & $13 \times 10^{-4}$ & $99.998 \%$ \\
\hline Sobel & 942 & 0 & 75855 & 3 & $39 \times 10^{-4}$ & $99.996 \%$ \\
\hline Prewitt & 944 & 0 & 75855 & 1 & $13 \times 10^{-4}$ & $99.998 \%$ \\
\hline Laplace & 938 & 0 & 75855 & 7 & $91 \times 10^{-4}$ & $99.990 \%$ \\
\hline
\end{tabular}


International Journal of Computer Science \& Engineering Survey (IJCSES) Vol.8, No.2, April 2017

Table 12. Object $2 \mathrm{MR}$ result for $90^{\circ}$

\begin{tabular}{|c|c|c|c|c|l|c|}
\hline \multicolumn{6}{|l|}{ Result for object 2 / orientation $: 90^{\circ}$} \\
\hline EDGES & TP & FP & TN & FN & MR (\%) & tp \\
\hline Canny & 884 & 1 & 75915 & 0 & $13 \times 10^{-4}$ & $99.998 \%$ \\
\hline Sobel & 882 & 0 & 75916 & 2 & $26 \times 10^{-4}$ & $99.997 \%$ \\
\hline Prewitt & 883 & 0 & 75916 & 1 & $13 \times 10^{-4}$ & $99.998 \%$ \\
\hline Laplace & 871 & 0 & 75916 & 13 & $169 \times 10^{-4}$ & $99.983 \%$ \\
\hline
\end{tabular}

Table 13. Object $2 \mathrm{MR}$ result for $135^{\circ}$

\begin{tabular}{|c|c|c|c|c|l|c|}
\hline \multicolumn{7}{|c|}{ Result for object 2 / orientation : $135^{\circ}$} \\
\hline EDGES & TP & FP & TN & FN & MR (\%) & tp \\
\hline Canny & 912 & 1 & 75887 & 0 & $13 \times 10^{-4}$ & $99.998 \%$ \\
\hline Sobel & 907 & 0 & 75888 & 5 & $65 \times 10^{-4}$ & $99.993 \%$ \\
\hline Prewitt & 903 & 0 & 75888 & 9 & $117 \times 10^{-4}$ & $99.988 \%$ \\
\hline Laplace & 904 & 0 & 75888 & 8 & $104 \times 10^{-4}$ & $99.989 \%$ \\
\hline
\end{tabular}

Table 14. Object 2 MR result for $180^{\circ}$

\begin{tabular}{|c|c|c|c|c|l|c|}
\hline \multicolumn{7}{|c|}{ Result for object 2 / orientation : $180^{\circ}$} \\
\hline EDGES & TP & FP & TN & FN & MR (\%) & tp \\
\hline Canny & 994 & 2 & 75804 & 0 & $26 \times 10^{-4}$ & $99.997 \%$ \\
\hline Sobel & 992 & 0 & 75806 & 2 & $26 \times 10^{-4}$ & $99.997 \%$ \\
\hline Prewitt & 990 & 0 & 75806 & 4 & $52 \times 10^{-4}$ & $99.994 \%$ \\
\hline Laplace & 981 & 0 & 75806 & 13 & $169 \times 10^{-4}$ & $99.983 \%$ \\
\hline
\end{tabular}

Table 15. Object 3 MR result for $45^{\circ}$

\begin{tabular}{|c|c|c|c|c|l|c|}
\hline \multicolumn{7}{|c|}{ Result for object 3 / orientation : $45^{\circ}$} \\
\hline EDGES & TP & FP & TN & FN & MR (\%) & tp \\
\hline Canny & 1458 & 14 & 75328 & 0 & $182 \times 10^{-4}$ & $99.981 \%$ \\
\hline Sobel & 1466 & 0 & 75328 & 6 & $13 \times 10^{-4}$ & $99.992 \%$ \\
\hline Prewitt & 1471 & 0 & 75328 & 1 & $13 \times 10^{-4}$ & $99.998 \%$ \\
\hline Laplace & 1466 & 0 & 75328 & 6 & $78 \times 10^{-4}$ & $99.992 \%$ \\
\hline
\end{tabular}

Table 16. Object $3 \mathrm{MR}$ result for $90^{\circ}$

\begin{tabular}{|c|c|c|c|c|l|c|}
\hline \multicolumn{7}{|c|}{ Result for object 3 / orientation : $90^{\circ}$} \\
\hline EDGES & TP & FP & TN & FN & MR (\%) & tp \\
\hline Canny & 1451 & 14 & 75335 & 0 & $182 \times 10^{-4}$ & $99.981 \%$ \\
\hline Sobel & 1460 & 0 & 75335 & 5 & $65 \times 10^{-4}$ & $99.993 \%$ \\
\hline Prewitt & 1456 & 0 & 75335 & 9 & $117 \times 10^{-4}$ & $99.988 \%$ \\
\hline Laplace & 1455 & 0 & 75335 & 10 & $130 \times 10-4$ & $99.986 \%$ \\
\hline
\end{tabular}


International Journal of Computer Science \& Engineering Survey (IJCSES) Vol.8, No.2, April 2017

Table 17. Object $3 \mathrm{MR}$ result for $135^{\circ}$

\begin{tabular}{|c|c|c|c|c|l|c|}
\hline \multicolumn{7}{|c|}{ Result for object 3 / orientation : $135^{\circ}$} \\
\hline EDGES & TP & FP & TN & FN & MR (\%) & tp \\
\hline Canny & 1445 & 4 & 75351 & 0 & $52 \times 10^{-4}$ & $99.994 \%$ \\
\hline Sobel & 1439 & 0 & 75351 & 10 & $130 \times 10^{-4}$ & $99.986 \%$ \\
\hline Prewitt & 1448 & 0 & 75351 & 1 & $13 \times 10^{-4}$ & $99.998 \%$ \\
\hline Laplace & 1438 & 0 & 75351 & 11 & $143 \times 10^{-4}$ & $99.985 \%$ \\
\hline
\end{tabular}

Table 18. Object $3 \mathrm{MR}$ result for $180^{\circ}$

\begin{tabular}{|c|c|c|c|c|l|c|}
\hline \multicolumn{7}{|c|}{ Result for object 3 / orientation : $180^{\circ}$} \\
\hline EDGES & TP & FP & TN & FN & MR (\%) & tp \\
\hline Canny & 1397 & 0 & 75402 & 1 & $13 \times 10^{-4}$ & $99.998 \%$ \\
\hline Sobel & 1397 & 0 & 75402 & 1 & $13 \times 10^{-4}$ & $99.998 \%$ \\
\hline Prewitt & 1355 & 0 & 75402 & 43 & $559.8 \times 10^{-4}$ & $99.944 \%$ \\
\hline Laplace & 1367 & 0 & 75402 & 31 & $403.6 \times 10^{-4}$ & $99.959 \%$ \\
\hline
\end{tabular}

In the following figures histograms $(6,7,8,9)$, the MR for all the used edges techniques are implemented when applied for the three used sharpeners (objects) for the orientations $\left(45^{\circ}, 90^{\circ}\right.$, $135^{\circ}, 180^{\circ}$ ), each histogram figure include the MR\% (Misclassification ratio \%) of using the edges types over the three objects for one orientation divided by $\left(10^{-4}\right)$ to make the differences be more clearly than the actual values. Table 19 includes the edges types used for the objects with all the orientation cases, this table is gave a pre-idea about the best edge technique which is detected the object and not effected with the orientation of it. The figures mentioned are also used with the tables above to compare and find the best edge operator used to detect the sharpeners.

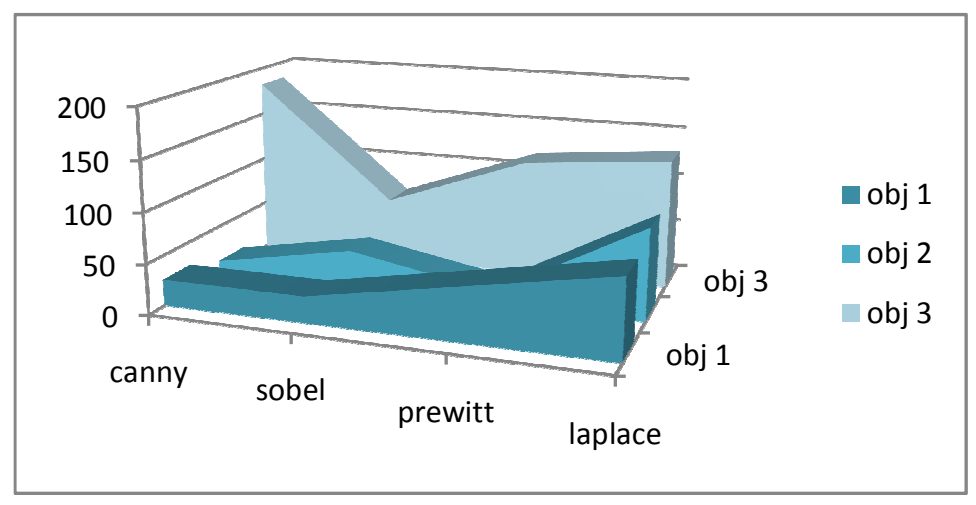

Figure. 6: Histogram for $45^{\circ} /\left(\mathrm{MR} \% / 10^{-4}\right)$ 
International Journal of Computer Science \& Engineering Survey (IJCSES) Vol.8, No.2, April 2017

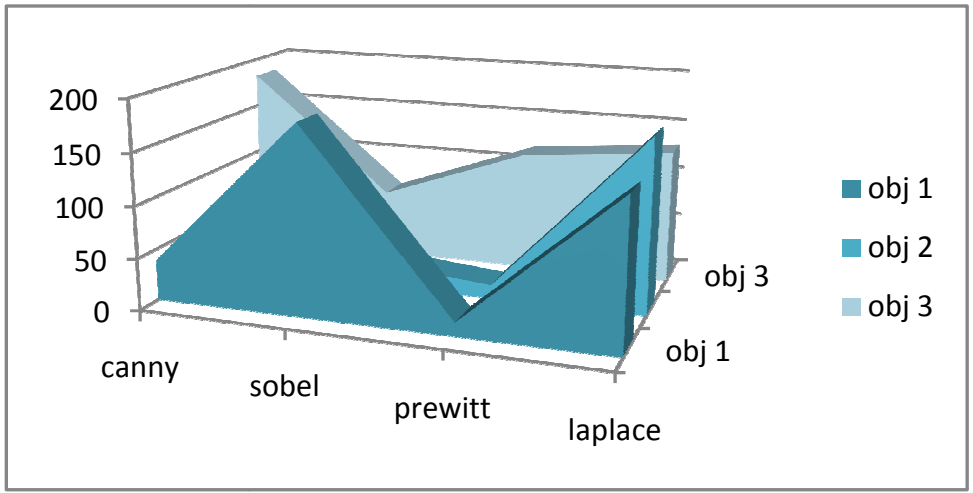

Figure 7: Histogram for $90^{\circ} /\left(\mathrm{MR} \% / 10^{-4}\right)$

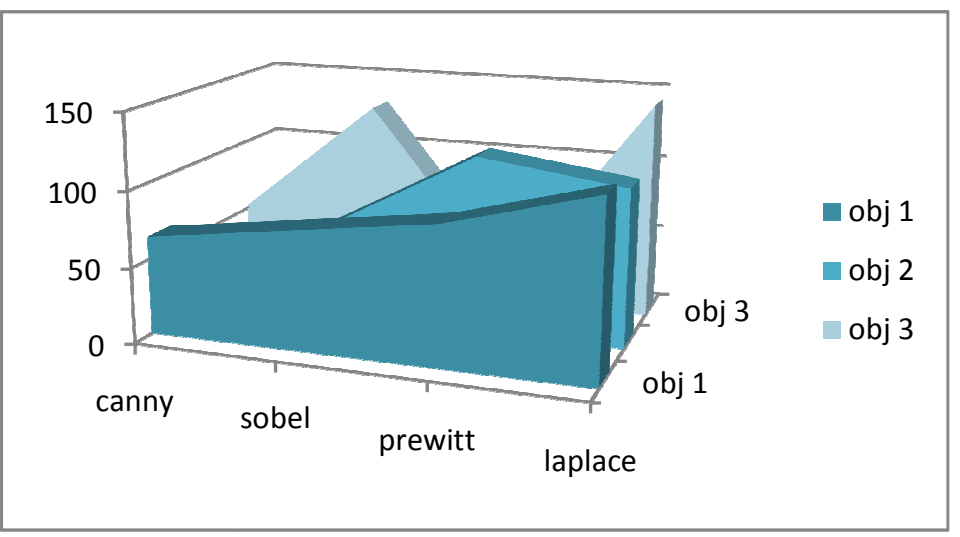

Figure 8: Histogram for $135^{\circ} /\left(\mathrm{MR} \% / 10^{-4}\right)$

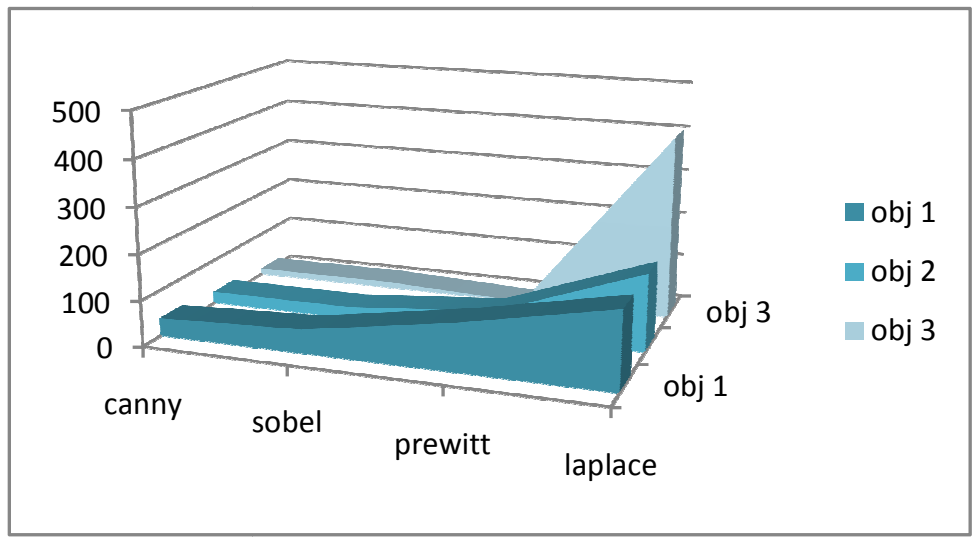

Figure 9: Histogram for $180^{\circ} /\left(\mathrm{MR} \% / 10^{-4}\right)$

Table 19. Best edge technique / orientation effectiveness

\begin{tabular}{|c|c|c|c|}
\hline & OBJ 1 & OBJ 2 & OBJ 3 \\
\hline $45^{\circ}$ & Canny & Canny & Sobel \\
\hline $90^{\circ}$ & Prewitt & Canny/Sobel & Sobel \\
\hline
\end{tabular}


International Journal of Computer Science \& Engineering Survey (IJCSES) Vol.8, No.2, April 2017

\begin{tabular}{|c|c|c|c|}
\hline $135^{\circ}$ & Canny & Canny & Prewitt \\
\hline $180^{\circ}$ & Canny & Canny/Sobel & Canny/Sobel \\
\hline
\end{tabular}

According to table 18 , the canny edge is the more remind type (8/12), the Sobel edge is the second mentioned type (5/12), while the Laplace operator is never remembered (the worse type) figures $(10,11,12,13)$ include a comparisons according to the tp rate. Whenever the MR is used, it is not important to uses both tp and fp rate, thus only the tp rate is implemented in this work inside the MR.

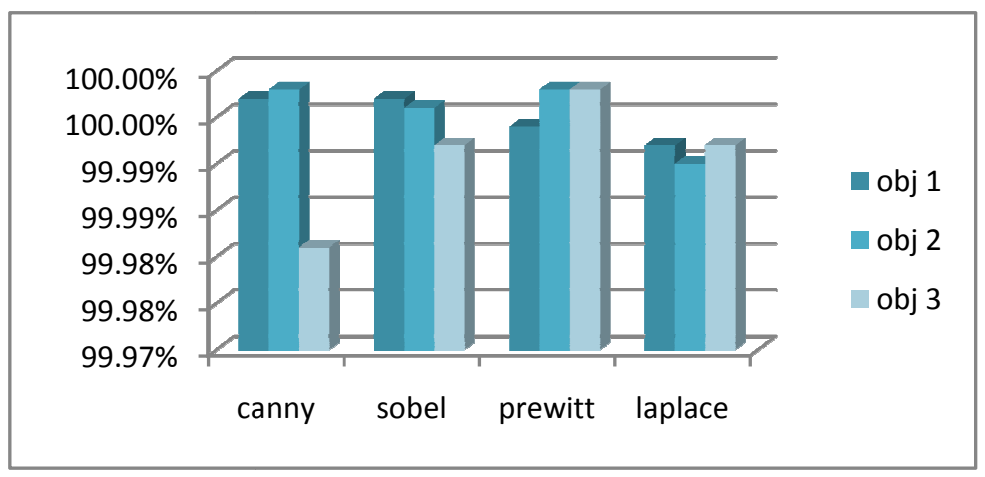

Figure 10: Histogram for $45^{\circ} /(t p \%)$

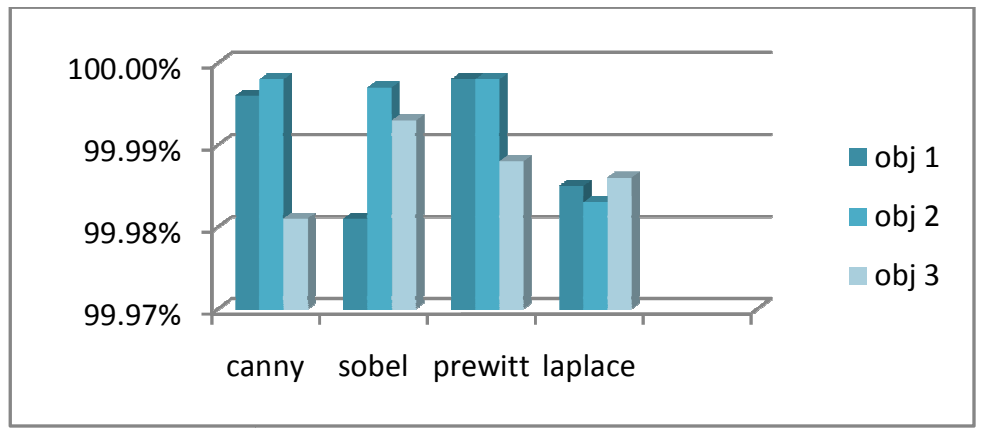

Figure. 11: Histogram for $90^{\circ} /(t p \%)$

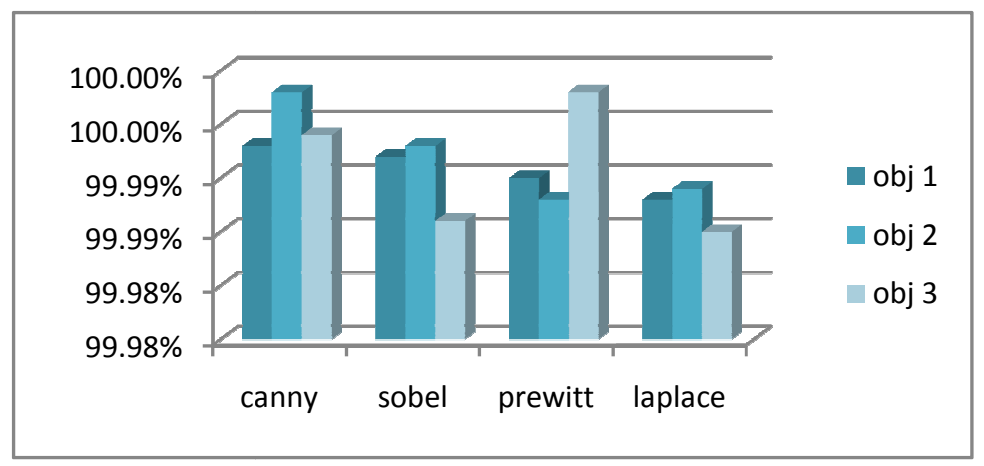

Figure 12: Histogram for $135^{\circ} /(t p \%)$ 


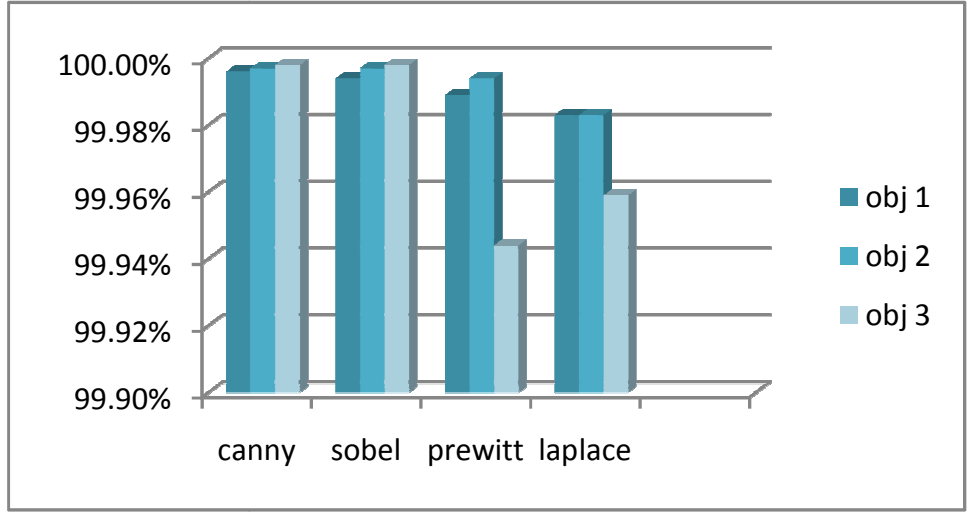

Figure 13: Histogram for $180^{\circ} /($ tp $\%)$

\section{Conclusion}

In this work, a study of orientation effectiveness is done for many edges detection techniques, the Canny, Sobel, Prewitt, and the Laplace operators are used to detect three different colored sharpeners, each one of these sharpeners are captured and detected in many orientation cases $(450,90 \mathrm{o}, 135 \mathrm{o}, 180 \mathrm{o}), \quad$ the results of detections using these detection types are compared one with each other for the all objects (sharpeners) under the orientations used during the camera capturing operation, in order to compare between the used edges detection techniques, the misclassification ratio, are used, the tp rate are then used inside the MR to ensure the given results, although that the Sobel is better than the other in two orientation used for the third sharpener (light green sharpener) but the average given results shown that the canny edge type is the best used one, the Laplace operator is the worst used technique for all the objects and the used orientations, finally and according to the results the canny edge is nominated to be used when an objects have to be detected or captured by camera under many expected orientation.

\section{FUTURE WORK}

Other type of complex objects 'shapes can be used inside the sharpeners. Insides the edges techniques, many other detection methods can be examined to check their orientations effectiveness. Other type of methods can be used inside the MR and the tp rate to find the best or the less effected method with the object orientation or effectiveness of the camera's orientation for the captured images.

\section{REFERENCES}

[1] Min C. Shin, Dmitry B. Goldgof, Kevin W. Bowyer, and Savvas Nikiforou, 2001. Comparison of Edge Detection Algorithms Using a Structure from Motion Task. IEEE Transactions on systems man, and cybernetics - part B: cybernetics, Vol. 31, No. 4.

[2] Syed Jahanzeb Hussain Pirzada, Ayesha Siddiqui. 2013. Analysis of Edge Detection Algorithms for Feature Extraction in Satellite Images IEEE International Conference on Space Science and Communication, Melaka, Malaysia.

[3] Andrew H. S. Lai, Member, IEEE, and Nelson H. C. Yung, Senior Member, IEEE, 2000. Lane Detection by Orientation and Length Discrimination, IEE transactions on systems, man and cybernetics, Vol. 30, No. 4.

[4] Viswanath Gopalakrishnan, Student Member, IEEE, Yiqun Hu, and Deepu Rajan, Member, IEEE, 2009. Salient Region Detection by Modeling Distributions of Color and Orientation 
International Journal of Computer Science \& Engineering Survey (IJCSES) Vol.8, No.2, April 2017

[5] Sherman Y. T. Lang, Member, IEEE, and Yili Fu, Member, IEEE, 2000. Visual Measurement of Orientation Error for a Mobile Robot, IEEE transactions on industrial and measurement, Vol. 49, No. 6.

[6] Umbaugh, Scott E 2010. Digital image processing and analysis : human and computer vision applications with CVIP tools (2nd ed.). Boca Raton, FL: CRC Press. ISBN 978-1-4398-0205-2.

[7] Canny ,J, 1986, A Computational Approach To Edge Detection, IEEE Trans. Pattern Analysis and Machine Intelligence, 8(6):679-698..

[8] Scharr, Hanno, 2000., Optimal Operators in Digital Image Processing. Interdisciplinary Center for Scientific Computing, Heidelberg University, Heidelberg, Germany.

[9] Gönen M., (2013), "Receiver Operating Characteristic (ROC) Curves", Memorial Sloan Kettering Cancer Center, Statistical and Data Analysis, Vol. 31, No. 210, pp. 1-18 .

[10] Fawcett T., (2005), "An Introduction to ROC Analysis", Pattern Recognition Letters, Institute for the Study of Learning and Expertise, Vol. 27, No. 8, pp. 861-874 .

[11] Irwin Sobel, (2014), "History and Definition of the so-called "Sobel Operator" more appropriately named the Sobel-Feldman Operator”, www.researchgate.net/publication/239398674.

[12] J. M. S. Prewit, "Object Enhancement and Extraction” Picture and Processing and Psychopictorics, B Lipkin and A Rosenfeld, EDS, New York: Academic Press, 1970, PP.. 75-149

\section{ACKNOWLEDGEMENTS}

I would like to thank my family for their help and support, the Mosul university staff, my friend Mothanna Osama kashmola for his support.

\section{AUTHOR}

Muhamad Azhar Abdilatef Al-Obaidy, Iraqi nationality, born in London -UK in 1984, he got the M.Sc. in computer engineering from Cankaya University, Ankara Turkey, 2014, B.Sc. in computer Engineering form Mosul University, Mosul , Iraq, 2006, Now, he is an assistant lecturer at college of engineering, university of Mosul, mechatronics engineering department, and also the head of Research and Development Department, Presidency of University of Mosul.

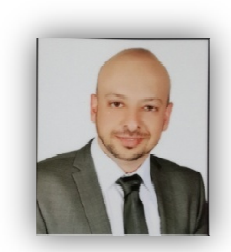

